\title{
ASSESSMENT OF ADVERSE DRUG REACTIONS OCCURRING AT DEPARTMENT OF NEUROLOGY OF A TERTIARY CARE HOSPITAL IN INDIA
}

\author{
JENNIFER R GRACE ${ }^{1}$, ANNA K SAINA ${ }^{1}$, MAHESWARI E ${ }^{2 *}$, SRINIVASA R ${ }^{3}$, VISWAM SUBEESH ${ }^{2}$
}

\begin{abstract}
${ }^{1}$ Department of Pharmacy Practice, M. S. Ramaiah College of Pharmacy, Bengaluru, Karnataka, India. ${ }^{2}$ Department of Pharmacy Practice, Faculty of Pharmacy, M. S. Ramaiah University of Applied Sciences, Bengaluru, Karnataka, India. ${ }^{3}$ Department of Neurology, M. S. Ramaiah Medical College and Hospitals, Bengaluru, Karnataka, India. Email: maheswarieswar@gmail.com
\end{abstract}

Received: 26 June 2018, Revised and Accepted: 12 July 2018

\section{ABSTRACT}

Objective: The purpose of this study was to assess the incidence and pattern of adverse drug reactions (ADRs) reported from the department of neurology of a tertiary care hospital in Karnataka, India.

Methods: It is a hospital-based prospective, observational study, conducted among the inpatients of all age groups of either sex for a period of 6 months. ADRs were reported by the clinical pharmacists and physicians of this hospital. ADRs obtained were categorized based on its causality, severity, preventability, predictability, and outcomes. Binary logistic regression was carried out to identify the predictors of ADR and Kaplan-Meier analysis was performed for survival analysis.

Results: A total of 250 patients were enrolled for the study in which 108 (43\%) patients were presented with at least one ADR and a total of 212 ADRs were observed. The highest rate of ADRs was observed with antiepileptics 61 (29.5\%). The most commonly reported ADRs were skin reactions 23 (10.8\%). Causality was assessed using three different scales which showed that most of the ADRs were probable. Severity, preventability, and predictability were assessed, of which 125 (59\%) ADRs were moderate, 192 (90.6\%) ADRs were probably preventable, and 156 (73.6\%) ADRs were predictable, respectively. The outcomes showed that 150 (70.1\%) patients recovered from the reactions. Predictors such as polypharmacy and duration of stay were found to be significant.

Conclusion: The study concluded that the prevalence of ADRs in the department of neurology is high. Thus, early detection and management of ADRs are essential to avoid further complications of the reaction.

Keywords: Adverse drug reactions, Neurology, Causality, Severity, Predictability, Preventability, Polypharmacy.

(c) 2018 The Authors. Published by Innovare Academic Sciences Pvt Ltd. This is an open access article under the CC BY license (http://creativecommons. org/licenses/by/4. 0/) DOI: http://dx.doi.org/10.22159/ajpcr.2018.v11i10.27737

\section{INTRODUCTION}

Clinical evolution of medications is a convoluted procedure which consumes both resources and time. During the diverse periods of clinical trials, the medications are tried for its transient safety and efficacy on a set number of deliberately chosen people. After the medication is affirmed for utilization by overall population, post-marketing surveillance helps to recognize the new adverse drug reactions (ADRs) related to the utilization of various medicated formulations [1]. An ADR is defined as "any response to a drug that is noxious and unintended, and that occurs at doses normally used in man for the prophylaxis, diagnosis, or therapy of disease, or for the modifications of physiological function" [2]. Monitoring of ADRs helps to evaluate the effectiveness and risk of medications, empower safe and rational use of drugs and enhance general patient care and well-being. The cost of ADRs in the community is high, and under-reporting of ADRs by health experts is a globally perceived issue. ADR identification and reporting may prevent the occurrence of ADRs and drug-related problems (DRPs) in future [1].

The patient may experience ADRs with single or multiple drugs as anticipated or may show up instantly, on continued use, even after cessation of therapy. Although ADRs influence all age groups, yet the most usually influenced ones are geriatrics and pediatrics. Geriatrics experience ADRs due to comorbid diseases, polypharmacy, altered pharmacokinetic, and pharmacodynamic changes which enhance hospital admissions. Pediatrics particularly neonates experience ADRs due to immature organ development and instabilities in pharmacokinetics and pharmacodynamics [2,3]. Administration of drugs acting on the central nervous system (CNS) during pregnancy may manifest potential teratogenic impacts on the fetus. Consumption of phenytoin increases the proportion of malformations such as orofacial clefts, cardiovascular deformities, and seizures. Medications such as carbamazepine, levetiracetam, and lorazepam can reach the fetus through breast milk and can harm them [4]. Hepatic and renal impairment with comorbidities, genetic polymorphisms, and medications with a narrow therapeutic index may demonstrate high occurrences of ADR.

Drugs acting on the CNS such as antiepileptic, antipsychotic, and anxiolytic contribute to ADRs such as extrapyramidal symptoms, insomnia, sedation, and even serious effects such as increasing suicidal tendency and depression [5]. Studies have showed that the patients in neurology department experience $23.5 \%$ of ADRs [6]. The ADRs transpire due to particular idiosyncratic mechanisms or harmful metabolic items which may lead to initial treatment failure [7]. ADRs can influence the consistence and course of treatment in patients exposed to drug therapy. The effect of ADRs can be determined based on cessation of therapy, treatment failure or due to an adverse event [8].

An active pharmacovigilance program is the need of the hour in all hospitals especially in Indian setup, as ADRs cause a significant burden to the patients and also to the economy. This project is aimed to work in this regard to monitor, detect, assess, and disseminate the ADRs which ensures patient safety and minimizes the cost of health care. Detection and monitoring of ADR in the inpatient department of neurology is lacking in Indian literature. The objectives of the study were to compare the incidence and pattern of ADRs, to detect and assess the causality, preventability, predictability, and severity of ADRs, to evaluate the management of ADR and to document and report the detected ADR. 
Thus, the current study improves the knowledge and pattern of ADRs in patients with neurological diseases.

\section{METHODS}

A hospital-based prospective, observational study was conducted among the inpatients of Department of Neurology of Ramaiah Memorial Hospital, Bengaluru, Karnataka for over a period of 6 months, from November 2016 to April 2017. Patients diagnosed with neurologic disorders and treated with one or more neurological agents were included in the study and screened for ADRs, irrespective of the neurologic diagnosis while samples with inadequate data and patients not willing to participate in the study were excluded. The ADRs that were identified and reported by clinical pharmacists and physicians were recorded. The data obtained include patient demographic details, relevant history, general and systemic examination, laboratory investigations, diagnosis, treatment, description and nature of reaction, date and time of onset of reaction, suspected drugs, severity, treatment offered, and outcomes. Permission from Institutional Ethical Committee of Ramaiah Medical College was obtained before instigation of the study.

The degree of association of drug and the adverse reaction is determined using three different scales, namely Naranjo's algorithm, World Health Organization (WHO) causality assessment scale, and Karch and Lasagna's causality assessment scale. Naranjo's algorithm involves a set of questionnaires, to each of which score has been provided ranging from -1 to +2 . The total score for a particular drug-ADR combination is calculated, and if it is $>9$ it is categorized as definite, 5-8 as probable, 1-4 as possible, and $<1$ as unlikely. WHO causality assessment scale and Karch and Lasagna's causality assessment scale are also used to determine causality. Modified Hartwig and Siegel severity assessment scales are used to determine the severity of the suspected ADRs. Severity of a reaction was determined based on the classification systems of the WHO and system of Hartwig et al. The severity of a reaction was classified as Mild, Moderate, and Severe which, in turn, is categorized into different levels of which Level 1 and 2 is categorized as Mild, Level 3, 4a, and 4b is categorized as Moderate, and Level 5, 6, and 7 is categorized as Severe. Mild reactions will be resolved over a period of time with or without an antidote and without extending the period of hospital stay. Moderate reactions are those which require specific treatment and hospitalizations for at least 1 day. Serious ADRs were considered as life-threatening leading to disability and required prolonged hospitalization usually in intensive care units. Preventability is measured using Modified Schumock and Thornton Preventability assessment scale. In this scale, it is categorized as definitely preventable, probably preventable, and not preventable based on a set of questions. Predictability of an ADR was done utilizing the Council of International Organization for Medical Sciences based on the incidence of the reactions reported in the available literature and the previous history of the reactions to the suspected drug under study [9].

\section{Statistical analysis}

Binary logistic regression was performed to identify the predictors contributing to the occurrence of ADR. Age, gender, the presence of comorbidities, route of administration, duration of stay, polypharmacy, diseases, and drugs were considered as the independent variable. $\mathrm{p} \leq 0.05$ was considered as statistically significant. We compared the survival function of the subjects (occurrence of ADR) with respect to polypharmacy by plotting the Kaplan-Meier (KM) graph. Mantel-Cox statistics was performed for the comparison of KM components.

\section{RESULTS}

\section{Percentage of occurrence of ADRs}

In our study, a total of 250 patients were enrolled and of which, 108 patients (43\%) were presented with at least one ADR (Fig. 1).

\section{Gender and age distribution based on ADR occurrence}

The male and female patients with or without ADRs were assessed. Of 78 females admitted $36(46.2 \%)$ were found to have at least one ADR and of 172 males, 72 (41.9\%) were found to have ADR (Fig. 2). Patients of all age groups were included in the study. Among patients between 0 and 19 years, 5 (50\%) patients showed a higher incidence of ADR followed by geriatrics in which 52 (48\%) of 107 experienced ADR (Table 1).

Correlation of occurrence of ADRs with comorbidities

Comparing patients with and without comorbidities, patients with comorbidities were found to have more ADRs $77(71.3 \%)$ when compared to patients without comorbidities 31 (28.7\%) (Table 2).

Correlation of occurrence of ADRs with number of drugs prescribed More number of ADRs were seen in patients with polypharmacy $(>5$ drugs). 95(54\%) patients experienced ADRs (Table 3).

\section{Route of administration of suspected drugs}

In our study, ADRs were commonly observed in orally administered drugs when compared to parenteral drugs (Fig. 3).

\section{Correlation of occurrence of ADR with duration of stay}

Correlation of occurrence of ADR with duration of stay was assessed, and the study revealed that occurrence of ADR prolonged the length of hospital stay (Table 4).

\section{Correlation of occurrence of ADR with time}

Delayed ADRs ( $>24 \mathrm{~h}$ ) were found to be more when compared to rapid ADRs (within $24 \mathrm{~h}$ ) (Fig. 4).

\section{Occurrence of ADRs in neurological disorders}

Patients diagnosed with epilepsy showed a higher percentage of incidences of ADRs when compared to other neurological disorders (Table 5).

Drug combinations involved in the pharmacokinetic and pharmacodynamic interaction

In the current study, 19 ADRs occurred due to pharmacokinetic and pharmacodynamic interactions, among which 11 reactions were due to pharmacodynamic interactions and 5 were due to pharmacokinetic interactions. The summary of the drug combinations involved in pharmacokinetic and pharmacodynamics is shown in Table 6.

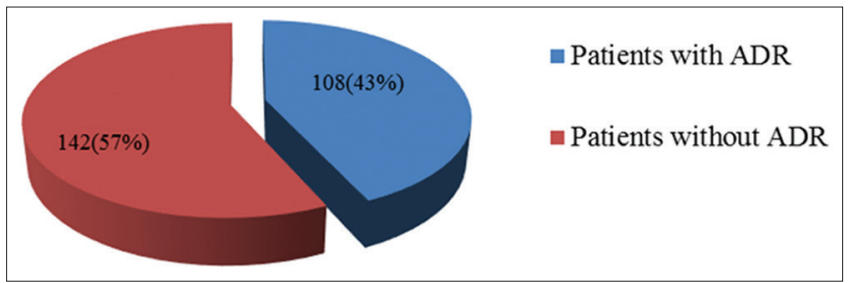

Fig. 1: Percentage of occurrence of adverse drug reactions

Table 1: Age distribution in patients with or without ADR

\begin{tabular}{|c|c|c|c|c|}
\hline \multirow[t]{2}{*}{ ADR } & \multicolumn{4}{|l|}{ n (\%) } \\
\hline & 0-19 years & 20-39 years & 40-59 years & $>60$ years \\
\hline Patients with ADR & $5(50)$ & $19(33.9)$ & $32(41.6)$ & $52(48.6)$ \\
\hline Total number of patients & $10(100)$ & $56(100)$ & $77(100)$ & $107(100)$ \\
\hline
\end{tabular}

ADR: Adverse drug reactions 
Table 2: Correlation of occurrence of ADRs with comorbidities

\begin{tabular}{lll}
\hline Comorbidities & n (\%) & \\
\cline { 2 - 3 } & $\begin{array}{l}\text { Patients with } \\
\text { ADR }\end{array}$ & $\begin{array}{l}\text { Patients without } \\
\text { ADR }\end{array}$ \\
\hline With comorbidities & $77(71.3)$ & $82(57.7)$ \\
Without comorbidities & $31(28.7)$ & $60(42.3)$ \\
Total & $108(100)$ & $142(100)$ \\
\hline
\end{tabular}

ADR: Adverse drug reactions

Table 3: Correlation of occurrence of ADRs with number of drugs prescribed

\begin{tabular}{llll}
\hline $\begin{array}{l}\text { Number of drugs per } \\
\text { prescription }\end{array}$ & $\mathbf{n}(\%)$ & & \\
\cline { 2 - 4 } & $\mathbf{2 - 3}$ drugs & 4-5 drugs & >5 drugs \\
\hline Patients with ADR & $3(15)$ & $10(18.5)$ & $95(54)$ \\
Patients without ADR & $17(85)$ & $44(81.5)$ & $81(46)$ \\
Total & $20(100)$ & $54(100)$ & $176(100)$ \\
\hline
\end{tabular}

ADR: Adverse drug reactions

Table 4: Correlation of occurrence of ADRs with duration of stay

\begin{tabular}{lll}
\hline Duration of stay (days) & $\mathbf{n}(\%)$ & \\
\cline { 2 - 3 } & $\mathbf{5 1 0}$ days & $>\mathbf{1 0}$ days \\
\hline Patients with ADR & $60(33.1)$ & $48(69.6)$ \\
Patients without ADR & $121(66.9)$ & $21(30.4)$ \\
Total & $181(100)$ & $69(100)$ \\
\hline
\end{tabular}

ADR: Adverse drug reactions

Table 5: Occurrence of ADRs in neurological disorders

\begin{tabular}{llll}
\hline Diseases & \multicolumn{2}{l}{$\mathbf{n}(\%)$} & \\
\cline { 2 - 4 } & $\begin{array}{l}\text { Patients } \\
\text { with ADR }\end{array}$ & $\begin{array}{l}\text { Patients } \\
\text { without ADR }\end{array}$ & Total \\
\hline Stroke & $64(43)$ & $85(57)$ & $149(100)$ \\
Epilepsy & $18(51.4)$ & $17(48.6)$ & $35(100)$ \\
Parkinson's disease & $4(44.4)$ & $5(55.6)$ & $9(100)$ \\
Meningitis & $8(80)$ & $2(20)$ & $10(100)$ \\
Headache & $1(10)$ & $9(90)$ & $10(100)$ \\
Others & $14(37.8)$ & $23(62.2)$ & $37(100)$ \\
\hline
\end{tabular}

ADR: Adverse drug reactions

\section{Category of drugs based on ADR occurrence}

Among the 212 ADRs observed, the highest rate of ADRs was observed in antiepileptics 61 (29.5\%) followed by antihypertensives 27 (13\%), anticoagulants 19 (9.2\%), antibiotics 15 (7.2\%), and antidiabetics $11(5.3 \%)$ (Table 7$)$

Among the commonly prescribed antiepileptics, levetiracetam $19(31.2 \%)$ was found to cause more number of ADRs followed by phenytoin $13(21.3 \%)$, carbamazepine $8(13.1 \%)$, gabapentin $6(9.9 \%)$, and oxcarbazepine 5 (8.2\%) (Table 8).

\section{Analysis of ADRs based on the system}

The organ system most commonly affected by ADRs were found to be gastrointestinal (GI) system 43 (20.3\%) followed by CNS $42(19.8 \%)$ and dermatological system $23(10.8 \%)$. The findings of the study showed that the most commonly reported ADR was found to be skin reactions $23(10.8 \%)$ followed by constipation $18(8.5 \%)$, giddiness $16(7.5 \%)$, hyponatremia $13(6.1 \%)$, tachycardia $11(5.2 \%)$, hypokalemia $10(4.7 \%)$, and headache 10 (4.7\%) (Table 9).

\section{Treatment of ADR}

All the reactions were managed appropriately in our study. In 94 reactions, either the suspected drug was withdrawn, and changes in

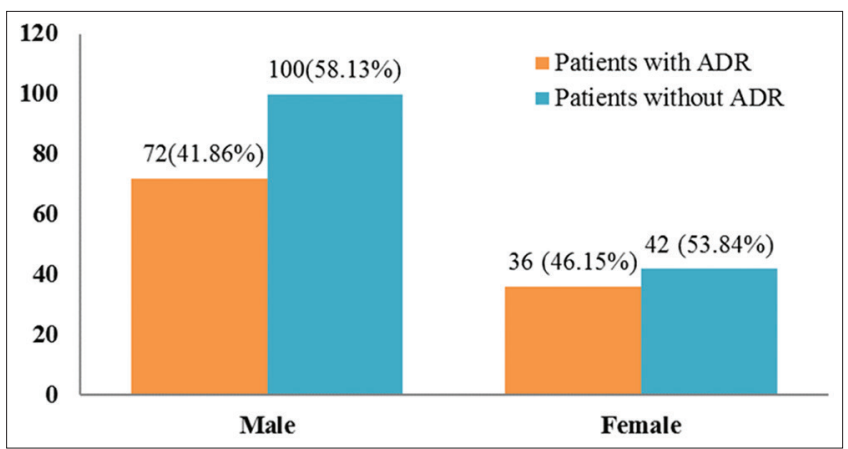

Fig. 2: Gender distribution based on adverse drug reactions occurrence

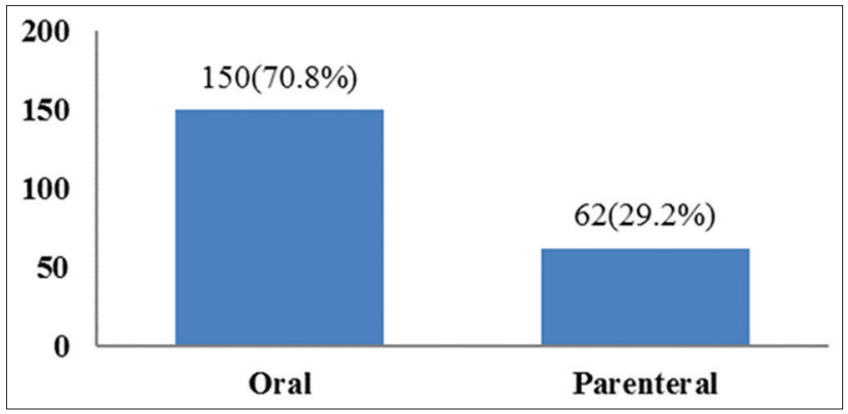

Fig. 3: Route of administration of suspected drugs

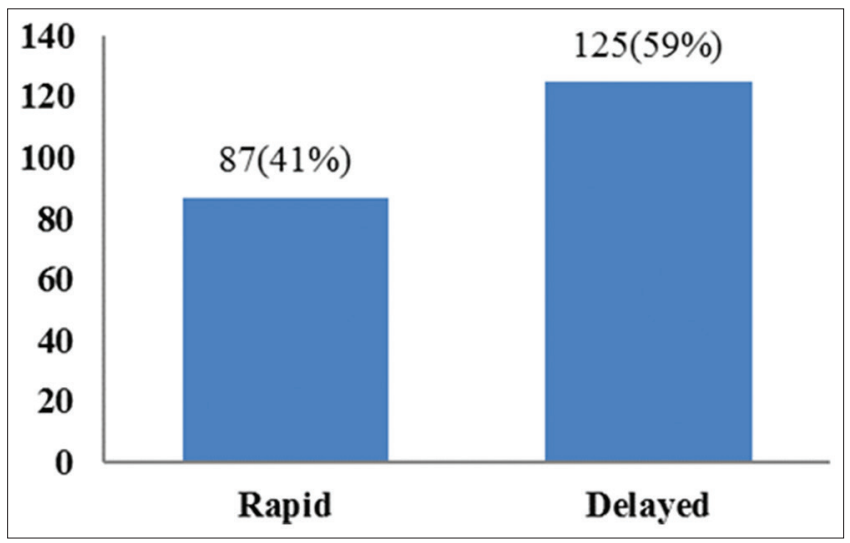

Fig. 4: Classification based on adverse drug reactions occurrence

doses were made, and in 111 reactions, specific antidote or treatment was given for the ADR. Continuous monitoring was done in 52 reactions to prevent the worsening of ADR (Fig. 5).

\section{Assessment of ADR}

The causality of ADR was assessed using three different scales, namely Naranjo's Causality assessment scale, WHO causality assessment scale, and Karch and Lasagna's causality assessment scale. Naranjo's Causality assessment scale indicated that 149 (70.3\%) ADRs were "probable" and $63(29.7 \%)$ ADRs were "possible." WHO causality assessment scale inferred that 142 (67\%) ADRs were "probable," 64 (30.2\%) ADRs were "possible," and 6 (2.8\%) ADRs were "unlikely." Karch and Lasagna's Causality scale showed that 145 (68\%) of the ADRs were probable followed by possible (Fig. 6). The severity of the reported reactions was assessed using Modified Hartwig and Siegel Severity Assessment Scale which showed that $125(59 \%)$ of the ADRs were categorized as moderate, $87(41 \%)$ as mild, and none of the ADRs were found to be severe. In moderate reactions, Level 3 contributed to the highest percentage 93 (43.9\%) (Fig. 7). The study revealed that 192 (90.6\%) of the ADRs were "probable preventable" followed by "not preventable" 16 (7.5\%) and "definitely preventable" 
Table 6: Drug combinations involved in pharmacokinetic and pharmacodynamics interaction

\begin{tabular}{lll}
\hline Drug combination & Interaction effect & Mechanism of interaction \\
\hline Enoxaparin+Clopidogrel+Aspirin & Hemorrhage & PD (Synergism) \\
Enoxaparin+Aspirin & Hemorrhage & PD (Synergism) \\
Glycopyrrolate+Ipratropium bromide & Anticholinergic effects & PD (Synergism) \\
Amantadine+Trihexyphenidyl & Anticholinergic effects & PD (Synergism) \\
Metoprolol+Telmisartan & Hyperkalemia & PD (Synergism) \\
Haloperidol+Ondansetron & ECG changes & PD (Synergism) \\
Nebivolol+Olmesartan+Hydrochlorothiazide & Hypotension & PD (Synergism) \\
Insulin+Metformin & Hypoglycemia & PD (Synergism) \\
Insulin+Telmisartan & Hypoglycemia & Unknown mechanism \\
Carbamazepine+Levetiracetam & Ataxia & Unknown mechanism \\
Carbamazepine+Levetiracetam & Dizziness & Unknown mechanism \\
Phenytoin+Clobazam & Ataxia & 1 \\
Phenytoin+Clobazam & Nystagmus & 1 \\
Phenytoin+Metronidazole & Dizziness & 1 \\
Phenytoin+Clopidogrel & Nystagmus & PK (metabolism) \\
\hline
\end{tabular}

Table 7: Category of drugs based on ADR occurrence

\begin{tabular}{|c|c|}
\hline Category: Suspected drugs & n (\%) \\
\hline $\begin{array}{l}\text { Antiepileptics (N03A): Phenytoin, Levetiracetam, Carbamazepine, Pregabalin, Oxcarbazepine, Clonazepam, Topiramate, Gabapentin, } \\
\text { and Sodium valproate }\end{array}$ & $61(29.5)$ \\
\hline Antibiotics (R02AB): Piperacillin+Tazobactam, Ceftriaxone, Linezolid, Ceftazidime, Meropenem, Tigecycline, and Cefuroxime & $15(7.2)$ \\
\hline $\begin{array}{l}\text { Antihypertensives (C02): Clonidine, Amlodipine, Metoprolol, Ramipril, Minoxidil, Mannitol, Telmisartan, Nebivolol, Losartan, } \\
\text { Hydrochlorothiazide, Olmesartan, Furosemide, and Atenolol }\end{array}$ & $27(13)$ \\
\hline Anticholinergics (R03BB): Tolterodine, Trihexyphenidyl, and Glycopyrrolate & $10(1.9)$ \\
\hline Antidiabetics (A10): Insulin, Metformin, Glimepiride, and Sitagliptin & $11(5.3)$ \\
\hline Analgesics (N02): Tramadol & $5(2.4)$ \\
\hline Lipid-modifying agents (C10): Atorvastatin & $2(1)$ \\
\hline Adrenergics, Inhalants (R03A): Salbutamol and Ipratropium bromide & $5(2.4)$ \\
\hline Hemorrhagic cystitis inhibitor (R05CB): Mesna & $5(2.4)$ \\
\hline Proton Pump Inhibitors (A02BC): Pantoprazole and Esomeprazole & $3(1.4)$ \\
\hline Antiemetics (A04A): Ondansetron & $6(2.9)$ \\
\hline Antineoplastics (L01): Rituximab & $2(1)$ \\
\hline Vasopressin antagonists (C03XA): Tolvaptan & $3(1.4)$ \\
\hline Cardiac glycoside (C01A): Digoxin & $1(0.5)$ \\
\hline Antiarrhythmics (C01B): Amiodarone & $1(0.5)$ \\
\hline Antigout preparations (M04A): Febuxostat & $2(1)$ \\
\hline Anesthetics, general (N01A): Propofol & $1(0.5)$ \\
\hline Thyroid Hormones (H03AA): Levothyroxine & $1(0.5)$ \\
\hline Antiarrhythmics (C01B): Amiodarone & $1(0.5)$ \\
\hline Antigout preparations (M04A): Febuxostat & $2(1)$ \\
\hline Iron preparations (B03A): Elemental Iron & $1(0.5)$ \\
\hline Antithrombotics agents (B01A): Dalteparin, Enoxaparin, Alteplase, Aspirin, Nicoumalone, Clopidogrel, and Cilostazol & $19(9.2)$ \\
\hline Antipsychotics (N05A): Lithium, Quetiapine, and Haloperidol & $5(2.4)$ \\
\hline Antifungals for systemic use (D01B): Voriconazole & $1(0.5)$ \\
\hline Vitamins (A11): Vitamin B & $1(0.5)$ \\
\hline Anesthetics, general (N01A): Propofol & $1(0.5)$ \\
\hline Thyroid Hormones (H03AA): Levothyroxine & $1(0.5)$ \\
\hline Antidepressants (N06A): Duloxetine and Venlafaxine & $5(2.4)$ \\
\hline Antiparkinson drugs (N04): Carbidopa, Levodopa, and Entacapone & $1(0.5)$ \\
\hline Antihistamines for systemic use (R06): Hydroxyzine and Chlorpheniramine & $2(1)$ \\
\hline
\end{tabular}

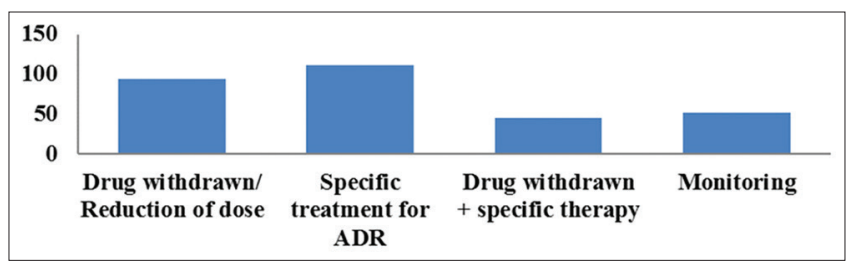

Fig. 5: Treatment of adverse drug reactions

4 (1.9\%) using Schumock and Thornton Preventability Assessment Scale (Fig. 8). The study suggests that majority of the ADRs were "predictable" $156(73.6 \%)$ when assessed by predictability scale (Fig. 9).

\section{Outcomes}

The outcomes of the suspected reactions were studied, and the results showed that $150(70.1 \%)$, of our study subjects recovered from the reactions whereas $51(24.1 \%)$ are still under recovery and $11(5.2 \%)$ of reactions were continuing. $5.2 \%$ of the reactions were estimated to be continuing of which $6(54.5 \%)$ reactions were mild and 5 (45.5\%) reactions were moderate (Fig. 10).

\section{Predictors of ADR}

Binary logistic regression was analyzed (Table 10) and the predictors contributing to the occurrence of ADR was evaluated. Age, gender, presence of comorbidities, route of administration, duration of stay, polypharmacy, diseases, and drugs are the contributing factors. In this 


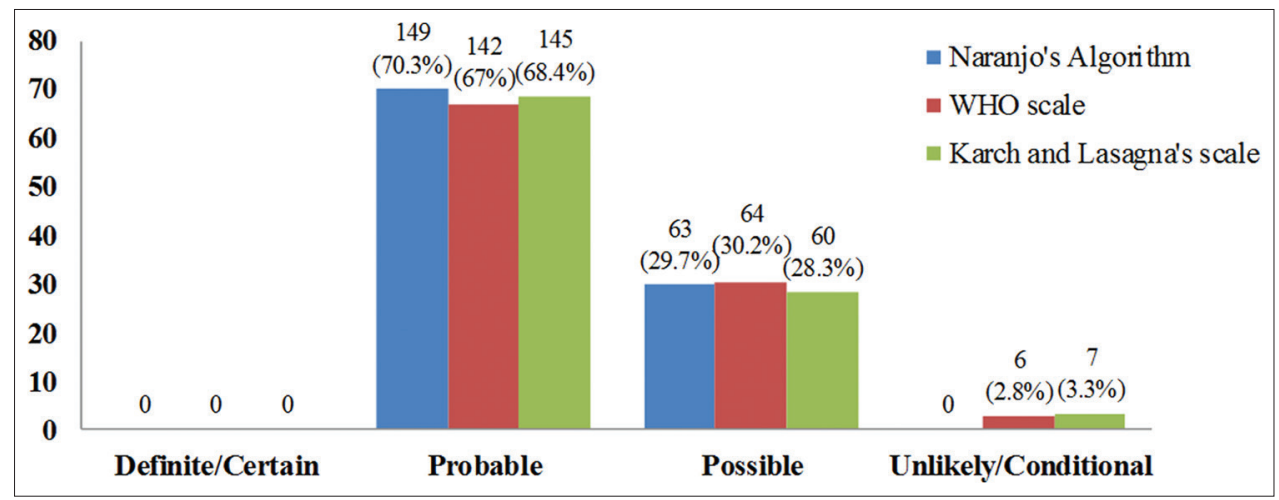

Fig. 6: Causality assessment scales

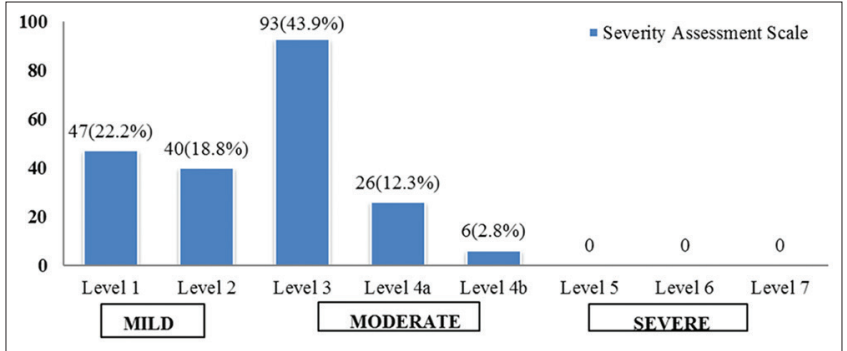

Fig. 7: Modified Hartwig and Siegel severity scale

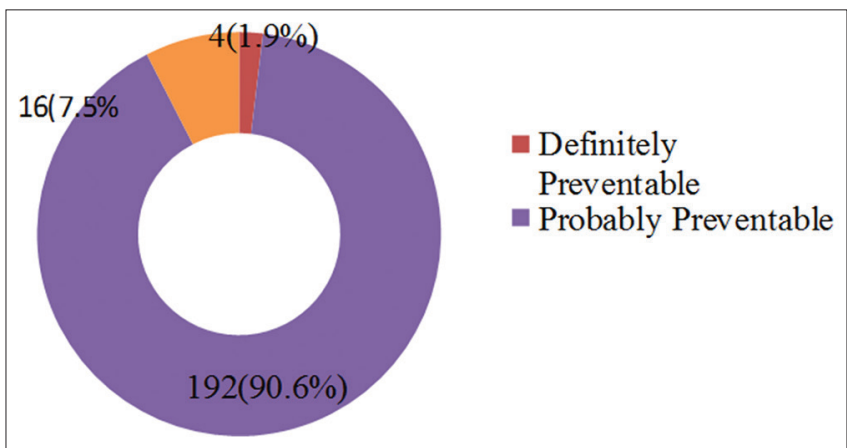

Fig. 8: Schumock and Thornton preventability scale

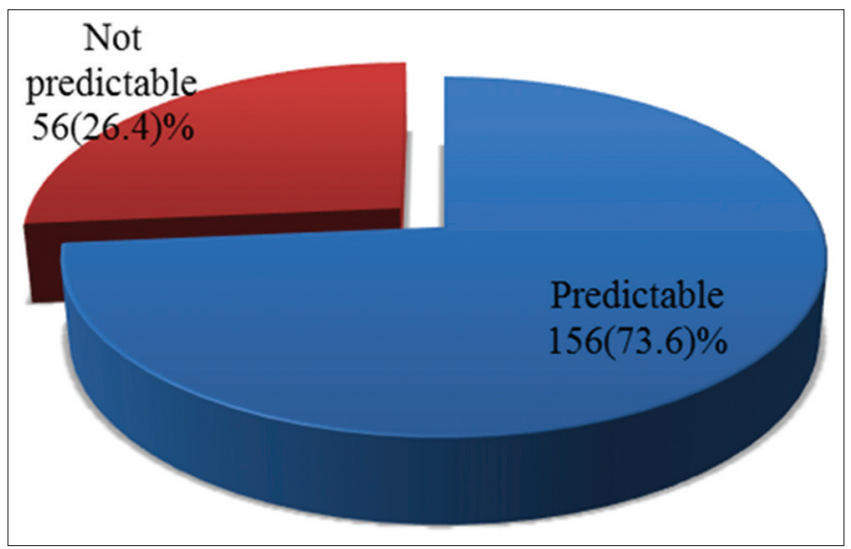

Fig. 9: Predictability scale

study, association was observed in patients with polypharmacy (odds ratio $-0.236,95 \%$ confidence interval $[\mathrm{CI}]$ 0.132-0.421, $\mathrm{p}=0.001$ ), duration of stay (odds ratio $-4.203,95 \%$ CI 2.323-7.602, $\mathrm{p}=0.001$ ), in diseases such as stroke (odds ratio - 1.811, 95\% CI 1.076-3.049,
Table 8: Occurrence of ADR in Antiepileptics

\begin{tabular}{ll}
\hline Antiepileptics & n (\%) \\
\hline Levetiracetam & $19(31.2)$ \\
Phenytoin & $13(21.3)$ \\
Carbamazepine & $8(13.1)$ \\
Gabapentin & $6(9.9)$ \\
Oxcarbazepine & $5(8.2)$ \\
Sodium Valproate & $3(4.9)$ \\
Fosphenytoin & $3(4.9)$ \\
Pregabalin & $2(3.3)$ \\
Topiramate & $1(1.6)$ \\
Clonazepam & $1(1.6)$ \\
Total & $61(100)$ \\
\hline
\end{tabular}

ADR: Adverse drug reactions

$\mathrm{p}=0.027$ ) and epilepsy (odds ratio - 3.976, 95\% CI 1.817-8.700, $\mathrm{p}=0.001$ ) in patients using levetiracetam (odds ratio - $1.952,95 \% \mathrm{CI}$ 1.096-3.477, $\mathrm{p}=0.027$ ) and with comorbidities (odds ratio - $1.817,95 \%$ CI 1.066-3.099, $\mathrm{p}=0.034$ ). Association was not observed in age, gender, and route of administration.

\section{KM analysis}

We compared the survival function of the subjects with respect to polypharmacy by plotting the KM graph. The KM graph displays the cumulative survival function on a linear scale by polypharmacy. The survival curve of no polypharmacy (labeled as " 0 ") was lower than that of with polypharmacy (labeled as "1"), which means that no polypharmacy has a higher probability of surviving (not experiencing an event, ADR) than polypharmacy (logrank statistics, $p=0.23$ ) (Table 11 and Fig. 11).

\section{DISCUSSION}

The current study is a hospital-based prospective and observational study conducted among the inpatients of department of neurology. Of the 250 patients reviewed, 108 (43\%) patients experienced at least one ADR. Among which 106 (98.1\%) patients experienced ADR during their hospital stay and $2(1.9 \%)$ patients were admitted due to the occurrence of ADRs. Our study showed an increase in the incidence of ADRs during the period of hospitalization which is in concordance with Nirojini et al., which stated that of total 295 patients, 157 patients experienced ADR during hospitalization [5].

In our study, a total of 172 male patients and 78 female patients were followed up of which 72 (41.9\%) male patients and $36(46.2 \%)$ female patients were presented with ADRs. Women were found to be affected with more number of ADRs when compared to men. This may be due to pharmacokinetic, pharmacodynamic, and hormonal changes in women. This finding is in agreement with Vijayakumar and Dhanaraju on the description of ADRs in a multi-specialty teaching hospital (2013) which expressed that more reports were observed 
Table 9: Organ system classification of ADRs

\begin{tabular}{|c|c|}
\hline System involved: Reactions & n (\%) \\
\hline $\begin{array}{l}\text { Gastrointestinal system disorders (0600): Loose stools (8), Constipation (18), Nausea and Vomiting (8), Gastric ulcer (1), Weight } \\
\text { loss (1), Abdominal pain (2), and Dry mouth (5) }\end{array}$ & $43(20.3)$ \\
\hline Central and peripheral nervous system disorders (0410): Giddiness, Somnolence, Dizziness (16), Ataxia (8), Seizure (3), & $42(19.8)$ \\
\hline Headache (10), Fever (2) Neuroleptic Malignant Syndrome (NMS) (1), Slurred speech (1), and Insomnia (1) & \\
\hline $\begin{array}{l}\text { Metabolic and nutritional disorders (0800): Hyponatremia (13), Hypokalemia (10), Hyperkalemia (2), Edema (9), Hypocalcemia (1), } \\
\text { and Lactic acidosis (1) }\end{array}$ & $36(17)$ \\
\hline $\begin{array}{l}\text { Skin and appendages disorders (0100): Drug Reaction Eosinophilia and Systemic Symptoms (DRESS) (1), Rash (14), Xerosis (4), } \\
\text { Erythema (4) }\end{array}$ & $23(10.8)$ \\
\hline $\begin{array}{l}\text { Heart rate and rhythm disorders (1030): Atrial fibrillation (1), Tachycardia with palpitations (11), ECG changes (2), and } \\
\text { Bradycardia (1) }\end{array}$ & $15(7.1)$ \\
\hline Red blood cell disorders (1210): Hemorrhage (7) and Anemia (7) & $14(6.6)$ \\
\hline Vision disorders (0431): Conjunctivitis (2), Nystagmus (7), and Ptosis (3) & $12(5.7)$ \\
\hline Respiratory system disorders (1100): Cough (6) & $6(2.8)$ \\
\hline Platelet, bleeding and clotting disorders (1230): Thrombocytopenia (3) and Thrombocytosis (1) & $4(1.9)$ \\
\hline Cardiovascular system disorders, general (1010): Hypotension (2) and Hypertension (2) & $4(1.9)$ \\
\hline Endocrine disorders (0900): Hyperglycemia (1), Hypoglycemia (2), and Cushings syndrome (1) & $4(1.9)$ \\
\hline Urinary system disorders (1300): Renal damage (2) and Urinary Tract Infection (UTI) (1) & $3(1.4)$ \\
\hline Reproductive disorders, male (1410): Erectile dysfunction (2) & $2(0.9)$ \\
\hline Musculoskeletal system disorders (0200): Rhabdomyolysis (1) & $1(0.5)$ \\
\hline Total (100) & $212(100)$ \\
\hline
\end{tabular}

ADR: Adverse drug reactions

Table 10: Binary logistic regression result of predictors associated with ADR

\begin{tabular}{|c|c|c|c|c|c|c|}
\hline \multirow[t]{2}{*}{ Factor } & \multirow[t]{2}{*}{ Odds ratio } & \multicolumn{2}{|l|}{ 95\% CI* } & \multirow[t]{2}{*}{ p value } & \multirow[t]{2}{*}{ Adjusted odds ratio } & \multirow[t]{2}{*}{$p$ value } \\
\hline & & Lower limit & Upper limit & & & \\
\hline \multicolumn{7}{|l|}{ Age (Years) } \\
\hline$\leq 55$ & 1.613 & 0.974 & 2.670 & 0.074 & - & \\
\hline$>55$ & Reference & & & & & \\
\hline \multicolumn{7}{|l|}{ Gender } \\
\hline Male & 1.190 & 0.695 & 2.040 & 0.582 & - & \\
\hline Female & Reference & & & & & \\
\hline \multicolumn{7}{|c|}{ Comorbidities } \\
\hline Yes & 1.817 & 1.066 & 3.099 & $0.034^{* *}$ & - & \\
\hline No & Reference & & & & & \\
\hline \multicolumn{7}{|c|}{ Route of administration } \\
\hline Oral & 0.592 & 0.322 & 1.088 & 0.113 & - & \\
\hline Parenteral & Reference & & & & & \\
\hline \multicolumn{7}{|c|}{ Polypharmacy (Drugs) } \\
\hline$>5$ & 0.236 & 0.132 & 0.421 & $0.001^{* *}$ & 0.161 & $0.001^{* *}$ \\
\hline$\leq 10$ & 4.203 & 2.323 & 7.602 & $0.001^{* *}$ & 0.288 & $0.001^{* *}$ \\
\hline$>10$ & Reference & & & & & \\
\hline \multicolumn{7}{|l|}{ Stroke } \\
\hline Yes & 1.811 & 1.076 & 3.049 & $0.027^{* *}$ & 18.409 & $0.001^{* *}$ \\
\hline No & Reference & & & & & \\
\hline \multicolumn{7}{|l|}{ Epilepsy } \\
\hline No & 3.976 & 1.817 & 8.700 & $0.001^{* *}$ & - & \\
\hline Yes & Reference & & & & & \\
\hline \multicolumn{7}{|c|}{ Levetiracetam } \\
\hline No & 1.952 & 1.096 & 3.477 & $0.027^{* *}$ & 2.809 & $0.013^{* *}$ \\
\hline Yes & Reference & & & & & \\
\hline \multicolumn{7}{|l|}{ Amlodipine } \\
\hline No & 1.560 & 0.915 & 2.661 & 0.104 & - & \\
\hline Yes & Reference & & & & & \\
\hline
\end{tabular}

${ }^{*}$ CI: Confidence interval, ${ }^{* *}$ Significance at 0.05

Table 11: Test of equality of survival distributions for the different levels of polypharmacy

\begin{tabular}{llll}
\hline & Chi-square & df & Sig. \\
\hline Logrank (Mantel-Cox) & 5.197 & 1 & 0.023 \\
\hline
\end{tabular}

in females (53.6\%) compared to males (46.4\%) [6]. Adolescents and geriatrics were most commonly affected with ADRs as observed in our study. This observation is contrary with the study of Palanisamy et al., on assessment, monitoring and reporting of ADRs in Indian hospital (2011) [10], where patients in the age group of 41-60 years $(53.33 \%)$ contributed to the highest percentage of ADRs. Patients with comorbidities were found to have more ADRs when compared to patients without comorbidities 77 (71.3\%). Maximum number of ADRs were seen in patients with polypharmacy which is in accordance with Ahmed et al. (2014) [11]. In our study, ADRs are commonly seen with orally administered drugs and delayed reactions were found to be more 


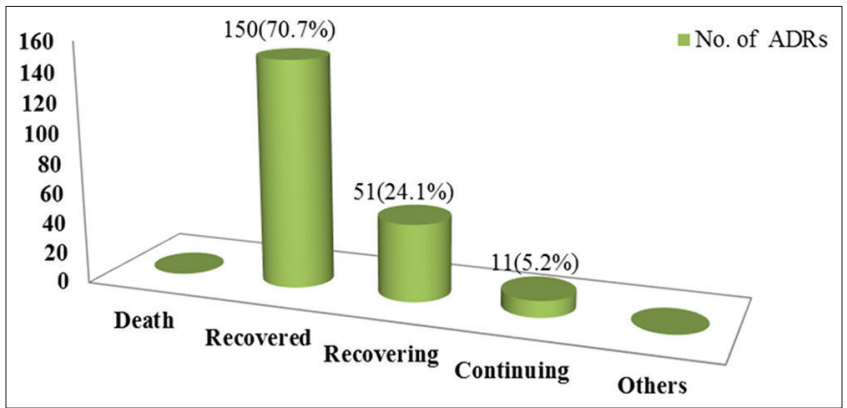

Fig. 10: Outcomes

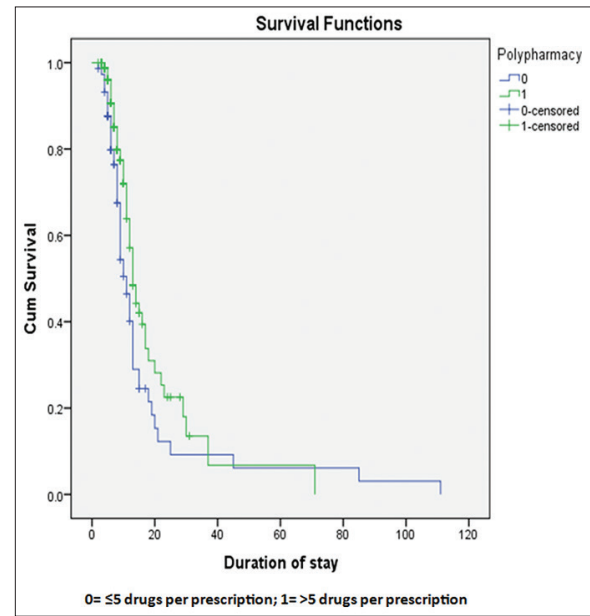

Fig. 11: Kaplan-Meier graph

which is in concordance with Lucca et al. The study finalized that the occurrence of ADR prolongs the length of hospital stay. 19 interactionled ADRs were observed among which 11 were pharmacodynamic and 5 were pharmacokinetic which is in par with Lucca et al., which stated that 27 (18.9\%) reactions were pharmacokinetic and 98 (68.9\%) reactions were pharmacodynamic [12].

The organ system most commonly affected by ADRs were found to be GI system 43 (20.3\%) followed by CNS 42 (19.8\%) which is in contrary with the study conducted by Nirojini et al. which observed that CNS (37.18\%) was most commonly affected followed by GI (14.07\%) [5]. The most commonly reported ADR was found to be skin reactions $23(10.8 \%)$ followed by constipation $18(8.5 \%)$ which is on par with the study conducted by Vijaykumar et al. [6]

Patients diagnosed with epilepsy showed a higher incidence of ADR when compared to other neurological disorders. The highest rate of ADRs was recorded to be induced by AEDs 61 (29.5\%) followed by antihypertensives 27 (13\%) which is in conformity with Thuermann et al., on detection of ADRs in a neurological department [13]. Among the antiepileptics, levetiracetam 19 (31.2\%) was found to cause more number of ADRs in contrary with Ramakrishna, on collection, detection, assessment, monitoring, and prevention of ADRs of antiepileptic drugs (2014) which inferred that phenytoin (44.7\%) caused more number of ADRs [7]. According to the literature, most of the antiepileptic drugs have a narrow therapeutic index, therefore, therapeutic drug monitoring has to be employed to ensure safe and effective treatment. Monotherapy and regular follow-up of patients on antiepileptics should be considered to prevent the adverse reactions and to improve the patient's compliance with drug therapy.

Among the total ADRs observed, 94 ADRs were managed either by withdrawing the suspected drug or by increasing or reducing the dose. Specific treatment along with or without a suitable antidote was given for 111 ADRs, to prevent further deterioration of reactions. This is contradictory to the study conducted by Vijayakumar et al. [14] which was conducted in intensive care and cardiac care units, where withdrawal of the drug was commonly initiated to manage the ADRs. In our study, administering an antidote or a specific treatment was the commonly adopted method. Abrupt cessation of the neurotropic drugs is not recommended as they lead to withdrawal complications; thus, dose has to be tapered slowly before it is withdrawn completely. Specific drug therapy has to be administered immediately on the appearance of an adverse reaction and/or an alternative can be prescribed.

Causality assessment scale showed that most of the ADRs were probably followed by possible. This study is in congruence with the study conducted by Singh et al., which illustrated that $36.36 \%$ were probably followed by possible with $31.16 \%$ [15]. The severity of the reported reactions was analyzed, and accordingly, 15 (59\%) ADRs were categorized as moderate, 87 (41\%) ADRs as mild and none of the ADRs were found to be severe. These findings were similar to the results of the study on Palanisamy et al. which confirmed that the majority of ADRs were moderate (58.33\%) followed by mild (35\%) [10]. The preventability assessment scale by Schumock and Thornton revealed that 192 (90.6\%) ADRs were "probable preventable" followed by 16 (7.5\%) "not preventable" and 4 (1.9\%) "definitely preventable." Majority of the ADRs were "predictable" 156 (73.6\%) as assessed by predictability scale. This study is similar to the study which is conducted by Raut et al., which proved that $45 \%$ of ADRs were "probably preventable" and 69\% were "predictable" [16].

The outcomes of the suspected reactions showed that $156(70.1 \%)$ study subjects recovered from the reactions. Severity and outcomes were correlated, most of the mild reactions showed faster recovery when compared to moderate reactions which were still under recovery. $11(5.2 \%)$ reactions were estimated to be continuing of which $6(54.5 \%)$ reactions were mild and $5(45.5 \%)$ reactions were moderate. These findings are in accordance with Vijayakumar and Dhanaraju where $77 \%$ recovered from reactions [6]. The early detection and management of adverse reactions can reduce health and economic burden on patients, and it also improves patients adherence and enhances health surveillance [17-19].

\section{CONCLUSION}

The study concludes that the prevalence of ADRs in the department of neurology is high. The literatures also support to this aspect, due to comorbidities and multiple drug regimens. Thus, early detection and management of ADRs are essential to avoid further progression of the reaction and its associated complications. Special attention should be provided to the patients who are at a higher risk of developing adverse reactions. Educational programs by clinical pharmacists will considerably reduce the complications thereby enhancing patient's adherence to the drug therapy in achieving better therapeutic outcomes. Future studies can be conducted for a longer period of time in both inpatients and outpatients to compare the pattern of ADRs. Prescribing pattern and other DRP could be studied to improve the safety profile.

\section{ACKNOWLEDGMENT}

We express our sincere gratitude to Dr. V. Madhavan, Professor, and Head, Department of Pharmacognosy, Faculty of Pharmacy, MSRUAS, for giving us an opportunity and for providing us all guidance and support.

\section{AUTHOR'S CONTRIBUTIONS}

Grace Jennifer Raju and Saina Anna Kurian: Data management, reporting, interpretation of results. E Maheswari: Developing an idea for the study, planning methodology, taking the responsibility for the implementation of the study. R Srinivasa: Providing guidance during the course of the study. Viswam Subeesh: Statistical analysis and interpretation of data. 


\section{CONFLICTS OF INTEREST}

There are no conflicts of interest.

\section{REFERENCES}

1. Rohilla A, Singh N, Kumar V, Kumar M, Sharma AD, Kushnoor A. Pharmacovigilance: Needs and objectives. J Adv Pharm Edu Res 2012;2:201-5.

2. Parthasarathi G, Olsson S. Adverse drug reactions. In: Parthasarathi G, Nyfort-Hansen K, Nahata MC, editors. A Textbook of Clinical Pharmacy Practice: Essential Concepts and Skills. $1^{\text {st }}$ ed. Chennai: Orient Longman PVT Ltd; 2004. p. 84-102.

3. Alam K, Shakya R, Ojha P. Reporting adverse drug reactions among hospitalized medical patients: A prospective study from tertiary care hospital in Western Nepal. Nepal J Epidemiol 2014;4:330-6.

4. Law R, Bozzo P, Koren G, Einarson A. FDA pregnancy risk categories and the CPS: Do they help or are they a hindrance? Can Fam Physician 2010;56:239-41.

5. Nirojini PS, Yemineni R, Nadenla RR. Monitoring and reporting of adverse drug reactions in a South Indian tertiary care hospital. Int $\mathbf{J}$ Pharm Sci Rev Res 2014;24:259-62.

6. Vijayakumar TM, Dhanaraju MD. Description of adverse drug reactions in a multi-speciality teaching hospital. Int J Integr Med 2013;1:1-6.

7. Ramakrishna P. Collection, detection, assessment, monitoring and prevention of adverse drug reactions of antiepileptic drugs. Int Res J Pharm 2014;5:653-7.

8. Singh G. Do no harm-but first we need to know more: The case of adverse drug reactions with antiepileptic drugs. Neurol India 2011;59:53-8.

9. Patel NH, Padhiyar J, Shah YB, Dixit RK. Study of causality, preventability and severity of cutaneous adverse drug reactions in a tertiary care institute. GCSMC J Med Sci 2015;4:24-7.

10. Palanisamy S, Kumaran KS, Rajasekaran A. A study on assessment, monitoring and reporting of adverse drug reactions in Indian hospital. Asian J Pharm Clin Res 2011;4:112-6.

11. Ahmed B, Nanji K, Mujeeb R, Patel MJ. Effects of polypharmacy on adverse drug reactions among geriatric outpatients at a tertiary care hospital in Karachi: A prospective cohort study. PLoS One 2014;9:e112133

12. Lucca JM, Ramesh M, Ram D, Kishor M. Incidence and predictors of adverse drug reactions caused by drug-drug interactions in psychiatric patients: An empirical study. Trop J Med Res 2016;19:29.

13. Thuermann PA, Windecker R, Steffen J, Schaefer M, Tenter U, Reese E, et al. Detection of adverse drug reactions in a neurological department: Comparison between intensified surveillance and a computer-assisted approach. Drug Saf 2002;25:713-24

14. Pal AB, Prusty SK, Sahu PK, Swain T. Drug utilization pattern of antiepileptic drugs: A pharmacoepidemiologic and pharmacovigilance study in a tertiary teaching hospital in India. Asian J Pharm Clin Res 2011;4:96-9.

15. Kumar BN, Nayak K, Singh H, Dulhani N, Singh P, Tewari P. A pharmacovigilance study in medicine department of tertiary care hospital in Chattisgarh (Jagdalpur), India. J Young Pharm 2010;2:95-100.

16. Raut AL, Patel P, Patel C, Pawar A. Preventability, predictability and seriousness of adverse drug reactions amongst medicine inpatients in a teaching hospital: A prospective observational study. Int J Pharm Chem Sci 2012;1:1293-8

17. Antony A, Joel JJ, Shetty J, Umar NF. Identification and analysis of adverse drug reactions associated with cancer chemotherapy in hospitalized patients. Headache. Int J Pharm Pharm Sci 2016;6:3-7.

18. Kumar L. Pharmacovigilance/reporting adverse drug reactions: An approach to enhance health surveillance and extending market share by minimizing the chances of drug withdrawals. Int J Pharm Pharm Sci 2015;7:1-7.

19. Kulkarni GP, Patil LV. Analysis of adverse drug reactions spontaneously reported to adverse drug monitoring centre of a tertiary care hospitalprospective study. Int J Curr Pharm Res 2018;10:23-5. 